\title{
Highly Selective Cyclooxygenase-1 Inhibitors P6 and Mofezolac Counteract Inflammatory State both In Vitro and In Vivo Models of Neuroinflammation
}

\author{
Rosa Calvello ${ }^{1 \dagger}$, Dario Domenico Lofrumento ${ }^{2 \dagger}$, Maria Grazia Perrone ${ }^{3+}$, \\ Antonia Cianciulli', Rosaria Salvatore', Paola Vitale ${ }^{3}$, Francesco De Nuccio', \\ Laura Giannotti ${ }^{2}$, Giuseppe Nicolardi ${ }^{2 *}$, Maria Antonietta Panaro ${ }^{1 *}$ and Antonio Scilimati ${ }^{3 *}$
}

'Department of Biosciences, Biotechnologies and Biopharmaceutics, University of Bari "A. Moro", Bari, Italy, ${ }^{2}$ Department of Biological and Environmental Sciences and Technologies, Section of Human Anatomy, University of Salento, Lecce, Italy, ${ }^{3}$ Department of Pharmacy - Pharmaceutical Sciences, University of Bari "A. Moro", Bari, Italy

Activated microglia secrete an array of pro-inflammatory factors, such as prostaglandins, whose accumulation contributes to neuronal damages. Prostaglandin endoperoxide synthases or cyclooxygenases (COX-1 and COX-2), which play a critical role in the inflammation, are the pharmacological targets of non-steroidal anti-inflammatory drugs, used to treat pain and inflammation. Since it was reported that COX-1 is the major player in mediating the brain inflammatory response, the aim of this study was to evaluate the effects of highly selective COX-1 inhibitors, such as P6 and mofezolac, in neuroinflammation models. Lipopolysaccharide (LPS)-activated mouse BV-2 microglial cells and LPS intracerebroventricular-injected mice as in vitro and in vivo neuroinflammation models, respectively, were used to probe the antiinflammatory efficacy of P6 and mofezolac. Both P6 and mofezolac reduce COX-1 expression in LPS-activated BV-2 cells. This reduction was accompanied with $\mathrm{PGE}_{2}$ release reduction and NF-kB activation downregulation. Coextensively, in the in vivo model, both glial fibrillary acidic protein and ionized calcium-binding adapter molecule-1 expression, two markers of inflammation, were reduced by mofezolac to a rank depending on the encephalon area analyzed. The increase of COX-1 expression observed in all the brain sections of LPS-treated mice was selectively downregulated by the in vivo treatment with mofezolac as well as $\mathrm{PGE}_{2}$ release and $\mathrm{Ik} \beta \alpha$ phosphorylation amount assayed in the brain areas tested. These results indicate the capability of P6 and mofezolac to modulate the NF-kB signaling pathway, emphasizing the neuroprotective effect and therapeutic potential of COX-1 inhibitors in the control of neuroinflammatory diseases.

Keywords: cyclooxygenase-1, P6 and mofezolac, cyclooxygenase-1 inhibitors, neuroinflammation, in vitro and in vivo experiments, lipopolysaccharide-treated BV2 microglial cells

\section{INTRODUCTION}

Neuroinflammation is widely recognized as an inflammatory response originated in the central nervous system (CNS). It is a pathological condition mainly caused by the nervous tissue infiltration of host defense cells and molecules from the bloodstream. In addition, it implies a complex interplay of glia, in particular microglia, typically associated with neurological and 
neurodegenerative diseases, triggering several concerns from a nosological viewpoint (1).

Microglia are recognized as the innate immune cells of the CNS, where they mediate a number of tissue homeostatic functions, including immune surveillance (2), synaptic regulation $(3,4)$, and neurogenesis $(5,6)$. Microglial activation and chronic inflammation thereafter is the starting point for elevated levels of a wide array of potentially neurotoxic molecules including proinflammatory cytokines, proteinases, and reactive oxygen species (ROS), which are believed to contribute to neurodegenerative processes (7-9).

Epidemiological data-based link between neuroinflammation and neurodegenerative diseases increased the worldwide scientific interest aimed to determine whether reducing inflammation would reverse neurodegeneration. Such data also indicate an inverse relationship between the use of traditional non-steroidal anti-inflammatory drugs ( $t$ NSAIDs) and Alzheimer's disease risk. $t$ NSAIDs pharmacological action is due to their ability to inhibit the cyclooxygenase (COX) and, hence, the biosynthesis of the prostaglandins (PG) involved in neuroinflammation (10).

Two COXs are known, COX-1 and COX-2. Upon inflammatory stimuli, COX-1, being constitutive in microglia, is responsible of the primary inflammatory response by inducing the production of PG, mainly $\mathrm{PGE}_{2}$. COX-2 is responsible, upon its induction, of a later and secondary response, with the exception of conditions in which the neurons are directly challenged (excitotoxicity and ischemia) (11). In the latter case, the primary response is COX-2 mediated, and microglia derived COX-1 succeeds as a secondary response upon perpetuating stimuli. In the first circumstances, selective COX-1 catalytic activity inhibition may result beneficial. $t$ NSAIDs inhibit COXs not selectively. Then, drugs useful to treat the different neuroinflammatory conditions are needed $(12,13)$.

Preliminarily, we accomplished an in vitro study using some selective COX-1 inhibitors (P6, P10, and SC-560) compared to aspirin and two selective COX-2 inhibitors (celecoxib and etoricoxib) in a in vitro model of neuroinflammation, represented by lipopolysaccharide (LPS)-stimulated N13 microglial cell line (14). In such a context, the treatment of LPS-stimulated microglial cells by selective COX-1 inhibitors P6 and P10 were able to downregulate COX-1 protein expression without affecting COX-2 levels; interfering with NF-kB activation (14).

In continuation of our investigations, herein, we report the validation of the study above mentioned by using both an in vitro model of neuroinflammation, represented by LPS-activated BV2 cell line, and an in vivo animal model, constituted by LPS-treated mice.

In particular, in this study, COX-1 role in neuroinflammation was explored by using P6 and mofezolac (Figure 1). Both compounds belong to the diarylisoxazole class, and mofezolac is the only clinically used diarylisoxazole approved in Japan as Disopain ${ }^{\circledR}$.

\section{MATERIALS AND METHODS}

\section{Reagents}

P6 was synthesized according to Di Nunno et al. (15), whereas mofezolac was synthesized following Micetich's protocol (16).

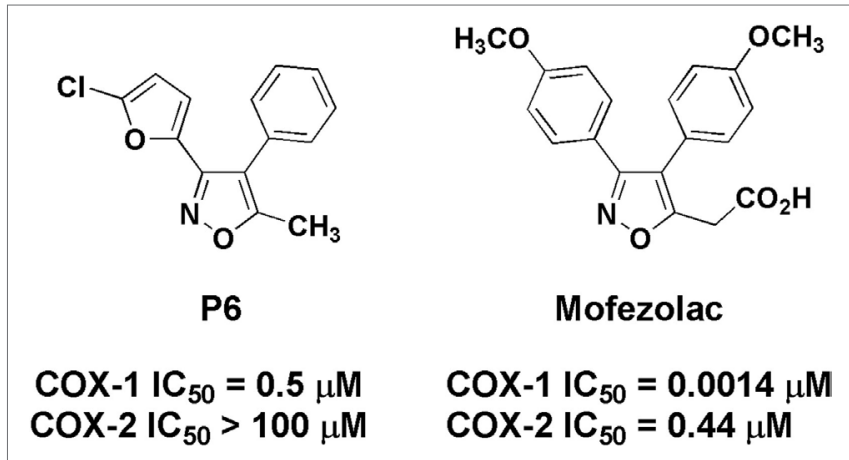

FIGURE 1 | Chemical structure of P6 and mofezolac. $I_{50}$ values refer to the human whole blood assay.

All the other reagents and solvents were purchased from SigmaAldrich (Milan, Italy) and used without any further purification.

Lipopolysaccharide from Escherichia coli serotype 0127: B8 was purchased from Sigma-Aldrich (Milan, Italy). The goat polyclonal a p-IкB (sc-7977) antibody (Ab) was purchased from Santa Cruz Biotechnology (DBA, Milan, Italy); COX-1 (Ab 695) and COX-2 (Ab 15191) Abs were obtained from Abcam (Cambridge, UK). Goat anti-rabbit IgG (sc-2004), goat antimouse IgG (sc-2005), and donkey anti-goat IgG (sc-2020) were purchased from Santa Cruz Biotechnology; mouse primary monoclonal antibody $(\mathrm{mAb})$ anti-glial fibrillary acidic protein (GFAP) (Merck Millipore, Milan, Italy), mouse mAb anti-ionized calcium-binding adapter molecule-1 (Iba-1) (Merck Millipore). Elisa kit for $\mathrm{PGE}_{2}$ evaluation was purchased from Cayman Chemical (Ann Arbor, MI, USA). MTT [3-(4,5-dimethylthiazol2-yl)-2,5-diphenyltetrazolium bromide], 3,3'-diaminobenzidine, and tribromoethanol were obtained from Sigma-Aldrich, Milan, Italy.

\section{Cell Cultures and Treatment}

BV2 microglia cells (ICLC HTL 03001-Interlab Cell Line Collection) were grown in high glucose Dulbecco's modified Eagle's medium supplemented with $10 \%$ fetal bovine serum, $100 \mathrm{U} / \mathrm{mL}$ penicillin, and $100 \mu \mathrm{g} / \mathrm{mL}$ streptomycin. They were maintained at $37^{\circ} \mathrm{C}$ in a humidified $5 \% \mathrm{CO}_{2} / 95 \%$ environmental air.

Then, microglial cells were plated, at a density of $25 \times 10^{4} /$ well in 6-well plates (Falcon) and treated with the chosen COX inhibitors (P6 and mofezolac) when they reached $80 \%$ confluence. Preliminary experiments were conducted to establish the optimal concentration and exposure times necessary for LPS $(1 \mu \mathrm{g} / \mathrm{mL})$ treatment, which were found to be in accordance with other reports $(17,18)$, as well as to establish the optimal dose of the COX inhibitors and exposure times to detect their effects on LPS-stimulated BV2 microglial cell function. In this regard, a set of experiments was carried out in which microglial cells were $1 \mathrm{~h}$ pretreated with the selective COX-1 inhibitors P6 (0.5 and $1.0 \mu \mathrm{M})$ or mofezolac $(0.1$ and $0.5 \mu \mathrm{M})$. Cells were then incubated for different times at $37^{\circ} \mathrm{C}$ with LPS, as pro-inflammatory stimulus. Experiments included cells grown in medium alone (control). 


\section{Cell Viability Test}

Cell viability of microglial cells was quantified using the MTT reduction assay. The cells $\left(8 \times 10^{3} /\right.$ well $)$ were grown in 96 -well plates (Becton Dickinson Labware) in complete medium and treated with different concentration of COX inhibitors, in presence or absence of LPS. Untreated cells were used as a control. A PBS $1 \times$ solution of MTT $(5 \mathrm{mg} / \mathrm{mL})$ was prepared and added to the cell medium at a final concentration of $0.5 \mathrm{mg} / \mathrm{mL}$. Cells were incubated for $4 \mathrm{~h}$ at $37^{\circ} \mathrm{C}$ and $5 \% \mathrm{CO}_{2}$ to allow the MTT metabolism. The formazan crystals formed (from MTT) into the cells were solubilized with DMSO (Sigma-Aldrich). The levels of MTT formazan were determined measuring the optical density at $\lambda=560 \mathrm{~nm}$ and subtracting the background $(\lambda=670 \mathrm{~nm}$ ) with a Victor Multiplate Reader (Wallac). Optical density was directly correlated to cell quantity.

\section{Immunoblotting Assay}

After treatment of cultures as previously described, cells were harvested and lysed by ice-cold lysis buffer [1\% Triton X-100, $20 \mathrm{mM}$ Tris- $\mathrm{HCl}, 137 \mathrm{mM} \mathrm{NaCl}, 10 \%$ glycerol, $2 \mathrm{mM}$ EDTA, $1 \mathrm{mM}$ phenylmethylsulfonyl fluoride, $20 \mu \mathrm{M}$ leupeptin hemisulfate salt, and $0.2 \mathrm{U} / \mathrm{mL}$ aprotinin (all from Sigma-Aldrich)] for 30 min on an ice bath.

Substantia nigra pars compacta, hippocampus, frontal lobe, and caudate from mice brains were minced in ice-cold PBS, washed, and then homogenized in a buffer containing lysis buffer $(50 \mathrm{mM}$ Tris $\mathrm{pH} 8,0.02 \mathrm{~g} / \mathrm{mL} \mathrm{NaCl}, 0.2 \%$ SDS, $1 \%$ Triton-X, $4 \mathrm{U} / \mathrm{mL}$ aprotinin, $2 \mathrm{mM}$ leupeptin, and $100 \mathrm{mM}$ phenylmethanesulfonyl fluoride).

The tissue and cell culture lysates were vortexed for 15-20 s and, then, centrifuged at $12,800 \times g$ for $20 \mathrm{~min}$. The protein concentration in the supernatant was spectrophotometrically determined by Bradford's protein assay. Protein samples were diluted with sample buffer $(0.5 \mathrm{M}$ Tris- $\mathrm{HCl} \mathrm{pH}=6.8,10 \%$ glycerol, $10 \%(\mathrm{w} / \mathrm{v})$ SDS, 5\% 2-mercaptoethanol, and $0.05 \%$ (w/v) bromophenol blue) and then boiled for $3 \mathrm{~min}$. Proteins $(25 \mu \mathrm{g} /$ lane) and prestained standards (BioRad Laboratories, Hercules, CA, USA) were loaded on $7 \%$ or $12 \%$ SDS precast polyacrylamide gels (BioRad Laboratories).

After electrophoresis, the resolved proteins were transferred from the gel to nitrocellulose membranes. A blotting buffer [20 mM Tris/150 mM glycine, $\mathrm{pH}=8.0$, and 20\% (v/v) methanol] was used for gel and membrane saturation and blotting. Then, membranes were incubated in the dark with the following specific primary Abs reported in reagents section for $60 \mathrm{~min}$ at room temperature. The membranes were washed with $0.1 \%$ Tween 20-PBS (for $20 \mathrm{~min}$, three times) and then incubated with the secondary Ab diluted 1:2,000 for 60 min. Bands were visualized by chemiluminescence detection (Invitrogen, Milan, Italy). The $\beta$-actin level was used as a protein loading control. For tissue analysis, obtained bands were normalized to the level of $\beta$-actin performed for each cerebral area tested. For cell cultures, the bands were normalized to the $\beta$-actin level of each experimental condition. The bands obtained after immunoblotting were submitted to densitometric analysis using 1D Image Analysis Software (Kodak Digital Science). Results were expressed as arbitrary units.

\section{Animals and Treatment Protocols}

This study was carried out in strict accordance with the European Council Directive 86/609/EEC and the Italian animal welfare legislation (art. 4 and 5 of D.L. 116/92). Seventy adult male 129S2/Sv mice (22-24 g body mass, 8-10 weeks of age) were purchased from Harlan-Italy and were kept under environmentally controlled conditions $\left(20 \pm 2{ }^{\circ} \mathrm{C}, 50-80 \%\right.$ humidity, $12 \mathrm{~h}$ light/dark cycle, food and water ad libitum) and divided into 7 groups of 10 animals ( 5 for western blotting and 5 for immunohistochemical procedures).

Mice received either the selective COX-1 inhibitor mofezolac (6 mg/kg, i.p.; indicated as $\mathrm{M}$ in all the figures) or vehicle (40\% DMSO in $0.1 \mathrm{M}$ phosphate buffer, $\mathrm{pH}=7.4$; $\mathrm{VM}$ in all the figures) once a day for 10 days. Mofezolac amount to be injected was chosen taken into account previous study and its COXs $\mathrm{IC}_{50}$ values. On the seventh day, mice were anesthetized with tribromoethanol $(250 \mathrm{mg} / \mathrm{kg}$, i.p.) and positioned in a stereotactic apparatus (Kopf Instruments, Tujunga, CA, USA). Vehicle (sterile saline, $5 \mu \mathrm{L}$; V-LPS in all the figures) or $5 \mu \mathrm{g}$ LPS in $5 \mu \mathrm{L}$ of sterile saline (LPS in all the figures) was administered into the cerebral lateral ventricle using a fine needle glass syringe (Hamilton, Lyon, France) and a syringe pump (KD Scientific, Holliston, MA, USA) at a rate of $1 \mu \mathrm{L} / \mathrm{min}$. This LPS dose and time point $(72 \mathrm{~h})$ induced the best neuroinflammatory response following a titration ( 24 up to $60 \mathrm{~h}$ ) preliminary study (data not shown). Stereotaxic injections coordinates were $2.3 \mathrm{~mm}$ dorsal/ventral, $1.0 \mathrm{~mm}$ lateral, and $0.5 \mathrm{~mm}$ anterior/posterior from the bregma. Mofezolac was given $30 \mathrm{~min}$ prior to LPS injection (M + LPS in all the figures).

\section{Immunohistochemical Staining}

Mice were transcardially perfused with tris-buffered saline $(\mathrm{pH}=7.6)$ followed by $4 \%$ paraformaldehyde in $\mathrm{PBS} \mathrm{pH}=7.4$ at $4^{\circ} \mathrm{C}$. Brains were subsequently postfixed in the same fixative, paraffin embedded, and $10 \mu \mathrm{m}$ slices were obtained with a rotative microtome (Leica, Milan, Italy). Immunohistochemistry was performed following a standard avidin-biotin complex procedure. Briefly, specimens were incubated with mouse primary $\mathrm{mAb}$ anti-GFAP at a ratio of 1:1,000 (Merck Millipore, Milan, Italy), or a mouse $\mathrm{mAb}$ anti-Iba-1 at a ratio of 1:500 (Merck Millipore) overnight at $4^{\circ} \mathrm{C}$ and then with an anti-mouse biotinylated secondary Ab (Dako, Milan, Italy), at a 1:1,000 dilution for $1 \mathrm{~h}$ at room temperature. The antigen-Ab complexes were visualized by sections incubation for $1 \mathrm{~h}$ with extravidin peroxidase (Sigma-Aldrich) diluted 1:1,500 and 3,3'-diaminobenzidine oxidation in the presence of $\mathrm{H}_{2} \mathrm{O}_{2}$.

\section{$\mathrm{PGE}_{2}$ Assay}

Microglial cells were cultured in 6-well plates at a density of $3 \times 10^{6}$ cells/well. Then, the cells were pretreated with selective COX-1 inhibitors P6 or mofezolac for $1 \mathrm{~h}$ and, subsequently stimulated with LPS $(1 \mu \mathrm{g} / \mathrm{mL})$. The cultures were maintained at $37^{\circ} \mathrm{C}$ for 24 and $48 \mathrm{~h}$ in a humidified air containing a $5 \% \mathrm{CO}_{2}$. $\mathrm{PGE}_{2}$ levels were determined in the supernatant using a competitive binding immunoassay (Cayman Chemical, Ann Arbor, MI, USA) following the manufacturer's instructions. Unstimulated cells were included as a control. $\mathrm{PGE}_{2}$ amount determination in 
the brain was performed in the tissue extracts, according to the manufacturer's instructions. The optical density was measured at $\lambda=405-420 \mathrm{~nm}$ with precision microplate reader and the amount of $\mathrm{PGE}_{2}(\mathrm{ng} / \mathrm{mL})$ was calculated using a $\mathrm{PGE}_{2}$ standard curve.

\section{Statistical Analysis}

Student's $t$-test and analysis of variance (one-way ANOVA) on the results of at least five independent biological replicates were performed. Values of $p<0.05$ were considered statistically significant.

\section{RESULTS}

\section{BV2 Microglial Cell Viability Assay}

MTT assay was used to quantitatively evaluate cell viability. This was performed to verify whether the tested selective COX-1 inhibitors (P6 and mofezolac) caused toxicity in LPS-treated BV2 cell line. Preliminarily, the effect of two different concentrations of P6 $(0.5$ and $1 \mu \mathrm{M})$ and mofezolac $(0.1$ and $0.5 \mu \mathrm{M})$ on BV2 microglial cell viability was evaluated. No cell toxicity was exerted by either P6, mofezolac, and LPS alone or a combination of LPS and each of the two inhibitors at $24 \mathrm{~h}$. The two concentrations of P6 and mofezolac were chosen based on the basis of previous studies and their COXs $\mathrm{IC}_{50}$ values (14).

Cell viability was found to be significantly $(p \leq 0.001)$ lower, after $48 \mathrm{~h}$ of LPS treatment (percentage cell viability equal to $78.4 \pm 0.15$ vs $99.8 \pm 0.07$ of the control), than untreated cells (control). None of the inhibitors used at the above indicated doses were toxic to BV2 microglial cells, when used in the absence of LPS (percentage cell viability ranging between $95.5 \pm 0.22$ and $98.2 \pm 0.16$ vs control). In addition, inhibitors used at the same doses, resulted protective toward BV2 microglial cells after $48 \mathrm{~h}$ LPS treatment $(1 \mu \mathrm{g} / \mathrm{mL})$, being the percentage of cell viability comparable to that observed in control.

\section{Evaluation of Mice Glial Activation}

Astroglial activation was characterized by immunoreactivity and immunoblotting analysis of the GFAP expression, a marker used to distinguish astrocytes from other glial cells of the CNS. LPS treatment determined an increase of immunoreactive cell bodies in comparison to untreated mice suggesting astrocyte activation in different brain regions. In particular, the caudate, frontal lobe, hippocampus, and substantia nigra were selected to evaluate the astrocyte activation after different animal treatment (Figures 2A-D). Images report GFAP reactivity in samples of mice control group (CTR), mice treated with LPS vehicle (V-LPS), LPS alone (LPS) or in combination with mofezolac ( $\mathrm{M}+\mathrm{LPS})$. In CTR sections, there is a physiological astroglial distribution with few immunoreactive elements surrounding the blood vessels as part of the brain-blood barrier, which means that there are no sign of reactive astrogliosis. In V-LPS-treated mice, the expression is similar to CTR, with perivascular immunoreactivity and the presence of a few astrocytes in the tissue, due to inflammation probably caused from the injection. In LPS-treated mice, images show a marked increase of the immunoreactive elements, whereas in vivo administration of mofezolac reduced the presence of GFAP immunoreactive cells in all the tested brain areas (Figures 2A-D).

Ionized calcium-binding adapter molecule-1 immunoreactivity, a marker of activated microglia, was also evaluated (Figure 3). LPStreated mice Iba-1 positive cells were more numerous, showing a more intense immunoreactivity, as well as a ramified phenotype, in comparison to untreated mice (Figures 3A-D). Mofezolac administration determined the reduction of Iba- 1 immunoreactivity in LPS-injected mice in all the tested brain areas (Figures 3A-D), suggesting that mofezolac reduces, at least in part, the microglial activation induced by the neurotoxic insult.

Immunoblotting analysis was also performed to semiquantitatively evaluate both astrocyte and microglia activation in samples derived from mice groups previously described. In LPS-treated mice, a significant increase of GFAP expression was detected in all the brain regions tested when compared to controls or vehicleLPS (Figure 4). Similar results were obtained for Iba-1 expression analysis (Figure 4).

Conversely, in mofezolac-treated animals previously injected with LPS, the GFAP as well as Iba-1 levels resulted significantly reduced in comparison to the animals that received LPS alone (Figure 4).

\section{Effect of the COX-1 Inhibitors -P6 and Mofezolac-on COXs Expression in BV2 Microglial Cell Line and Mice Brains}

The effect of mofezolac and P6 on COX-1 expression in LPStreated microglial cells was evaluated by western blotting analysis. After $24 \mathrm{~h}$, no significant difference between LPS-stimulated cells in the presence or absence of both COX-1 inhibitors was observed (data not shown). Interestingly, LPS-stimulated BV2 cells exhibited, at $48 \mathrm{~h}$, increased levels of COX-1 expression in comparison to untreated cells (Figure 5). The COX-1 expression levels evaluated at $48 \mathrm{~h}$ of incubation with LPS was significantly reduced in $1 \mathrm{~h}$ pretreated cells with either selective COX-1 inhibitor P6 or mofezolac (Figure 5). Therefore, COX-1 expression was found to be time dependently reduced by both selective inhibitors tested.

The expression of COX-2 in the same cell lysates was also evaluated. Interestingly, COX-2 protein levels were unaffected by the presence of $\mathrm{P} 6$ or mofezolac. In these conditions, COX-2 expression resulted comparable to the level observed in cells stimulated with LPS alone (Figure 5).

Therefore, in LPS-treated BV2 microglial cell line, P6 and mofezolac were able to reduce COX-1 expression without affecting COX-2.

Cyclooxygenases expression was also in vivo evaluated, assaying different brain regions (Figure 4). LPS-challenged mice exhibited increased levels of COX-1 both in comparison to controls and vehicle of LPS (vLPS) administered animals in all the different areas tested (Figure 4). Interestingly, LPS-challenged mice that received mofezolac treatment exhibited a significantly reduction of COX-1 expression in all brain areas tested. Protein levels comparable to controls were detected in mice treated with 


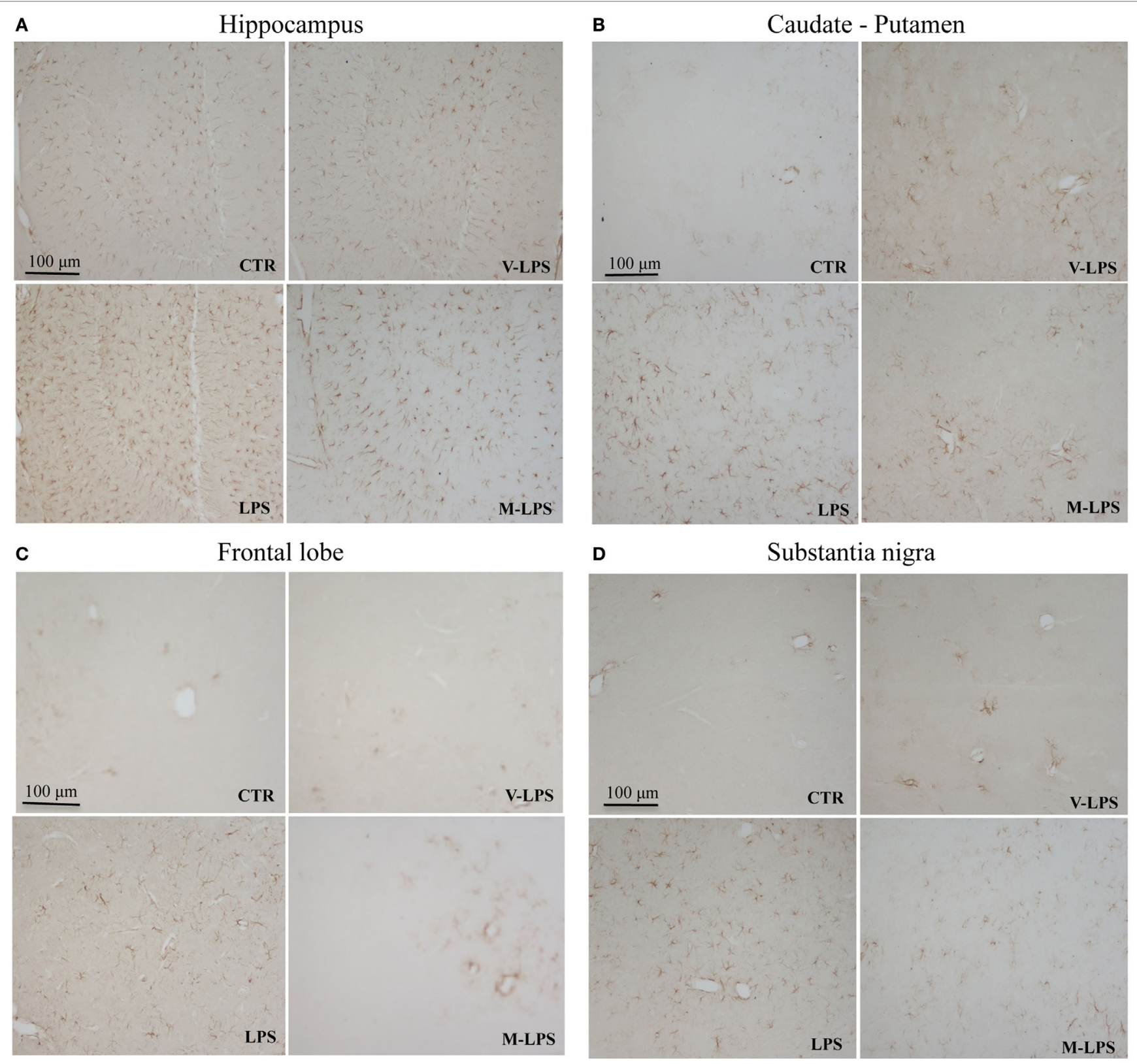

FIGURE 2 | Glial fibrillary acidic protein immunoreactivity in the hippocampus (A), caudate-putamen (B), frontal lobe (C), and substantia nigra (D), in slices of control (CTR), vehicle of LPS (V-LPS), lipopolysaccharide (LPS), mofezolac and LPS (M-LPS)-treated mice.

vLPS + mofezolac, whereas in mice treated with the vehicle of mofezolac + LPS, COX-1 levels resulted comparable to those detected in LPS-treated mice (Figure 4).

Immunoblotting assay on tissues of the same brain areas was performed to test the in vivo effects of mofezolac administration on COX-2 expression extent. COX-2 resulted overexpressed in all tested brain regions in LPS-administrated mice. Interestingly, mofezolac exhibited no effect on COX-2 modulation in LPStreated mice, being the protein levels almost comparable to LPS-treated mice (Figure 4).

\section{Effect of COX-1 Inhibitors on PGE Biosynthesis in BV2 Microglial Cells and Mouse Brain}

The PGE $\mathrm{P}_{2}$ biosynthesis extent was evaluated in supernatants of cell cultures at $48 \mathrm{~h}$ incubation time (Table 1A). $\mathrm{PGE}_{2}$ production in LPS-stimulated cells was significantly higher than its basal level present in the untreated cells (control). Interestingly, both COX-1 inhibitors were able to reduce in a dose-dependent manner $\mathrm{PGE}_{2}$ release in LPS-treated cells (Table 1A). 


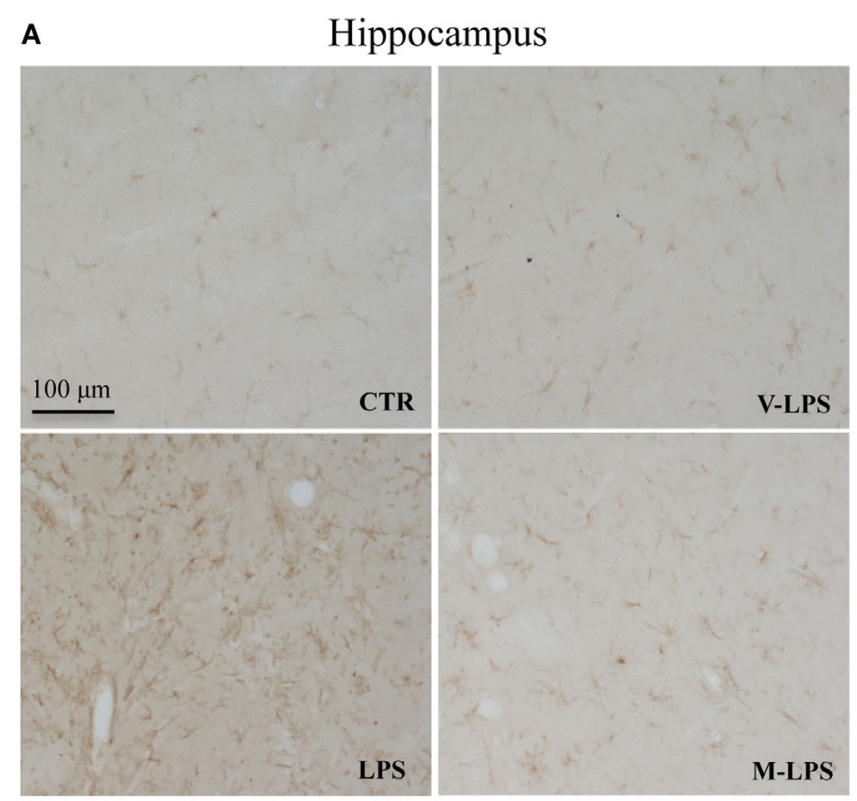

B

Caudate - Putamen

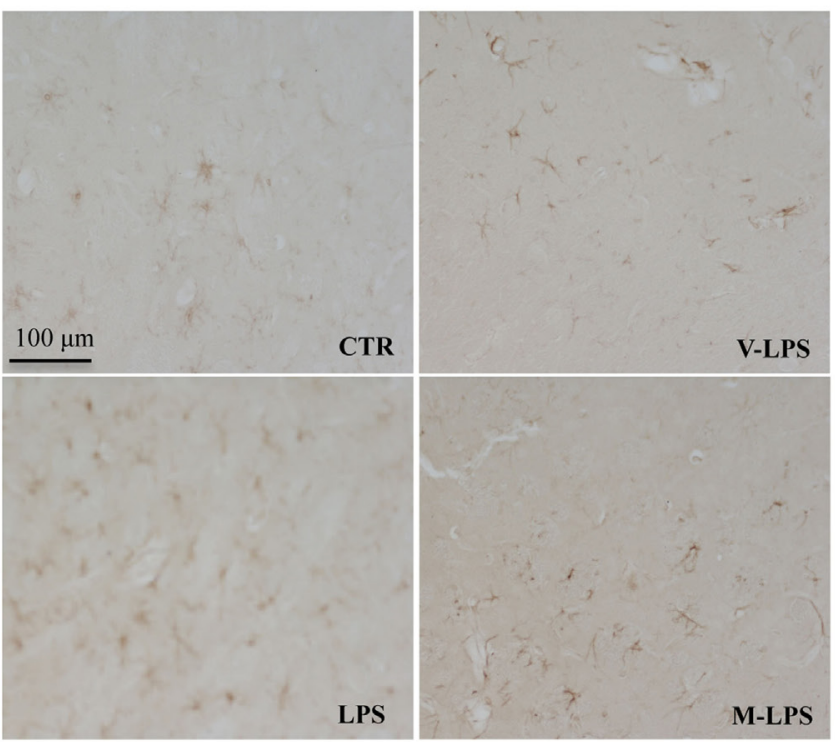

C

Frontal lobe

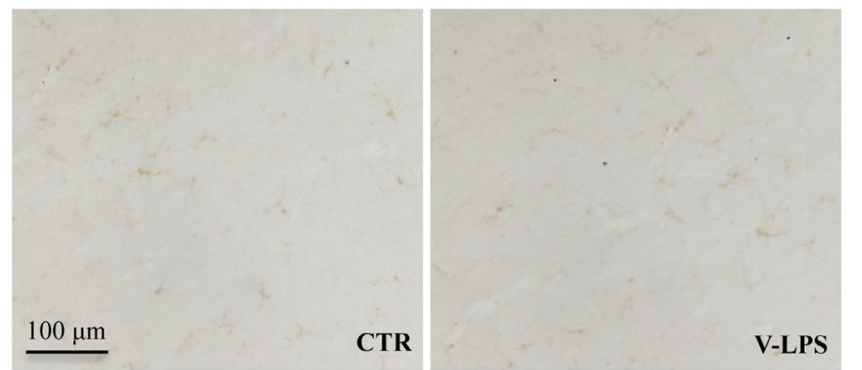

D

Substantia nigra

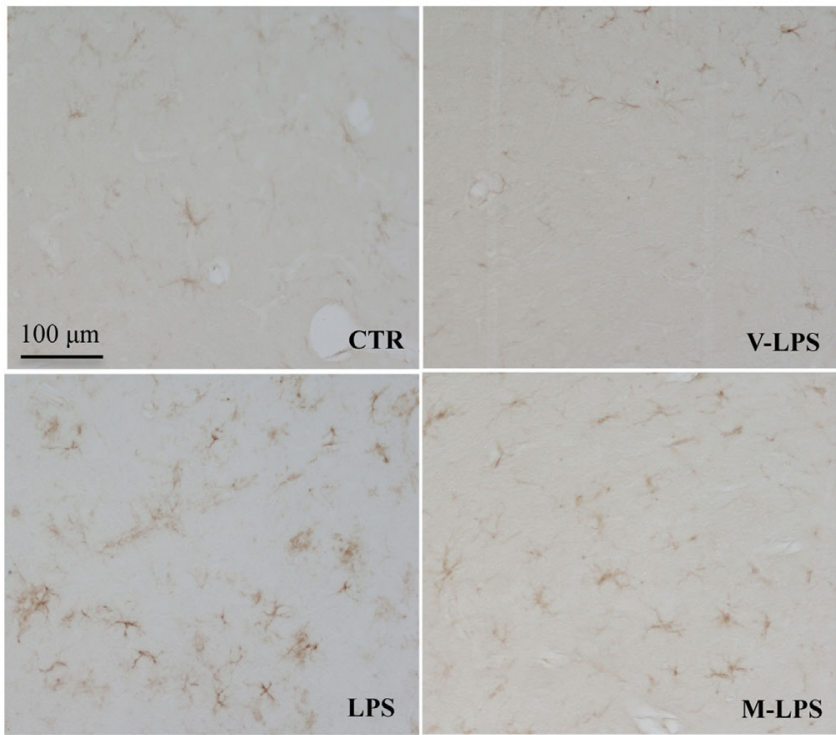

FIGURE 3 | lonized calcium-binding adapter molecule-1 immunoreactivity in the hippocampus (A), caudate-putamen (B), frontal lobe (C), and substantia nigra (D), in slices of control (CTR), vehicle of LPS (V-LPS), lipopolysaccharide (LPS), and mofezolac and LPS (M-LPS)-treated mice.

In the tested brain regions of LPS-treated mice, $\mathrm{PGE}_{2}$ levels were significantly increased with respect to control animals or mice receiving the vLPS (Table 1B). Interestingly, a significant reduction of $\mathrm{PGE}_{2}$ levels was detected in mofezolac-treated mice before LPS challenging (Table 1B).

\section{Effect of COX-1 Inhibitors on NF-kB Activation in LPS-Treated BV2 Microglial Cells and Mouse Brain}

Since the phosphorylation and degradation of $\mathrm{I} k \mathrm{~B} \alpha$, the inhibitory complex of NF-kB, is an essential step for NF-kB activation, the expression levels of the phosphorylated form of $\mathrm{I} k \mathrm{~B} \alpha(\mathrm{p}-\mathrm{I} k \mathrm{~B} \alpha)$ were evaluated. In this context, BV2 microglial cells exposed to LPS exhibited a significant increase of $\mathrm{p}-\mathrm{I} k \mathrm{~B} \alpha$ in comparison with no stimulated cells (control) (Figure 5). Densitometric analysis revealed little $\mathrm{p}-\mathrm{I} k \mathrm{~B} \alpha$ in the untreated cells, whereas pretreatment with all tested COX- 1 inhibitors, significantly reduced $\mathrm{I} k \mathrm{~B} \alpha$ phosphorylation in LPS-stimulated cells (Figure 5).

Similar results were obtained in in vivo model. In fact, in all tested brain areas of LPS-treated mice, an increase of $\mathrm{p}-\mathrm{I} k \mathrm{~B} \alpha$, in comparison to control animals as well as to vLPS administered mice, was detected. Conversely, in mice receiving mofezolac (M), a significant reduction of $\mathrm{p}-\mathrm{I} k \mathrm{~B} \alpha$ in all tested areas was detected (Figure 4). 

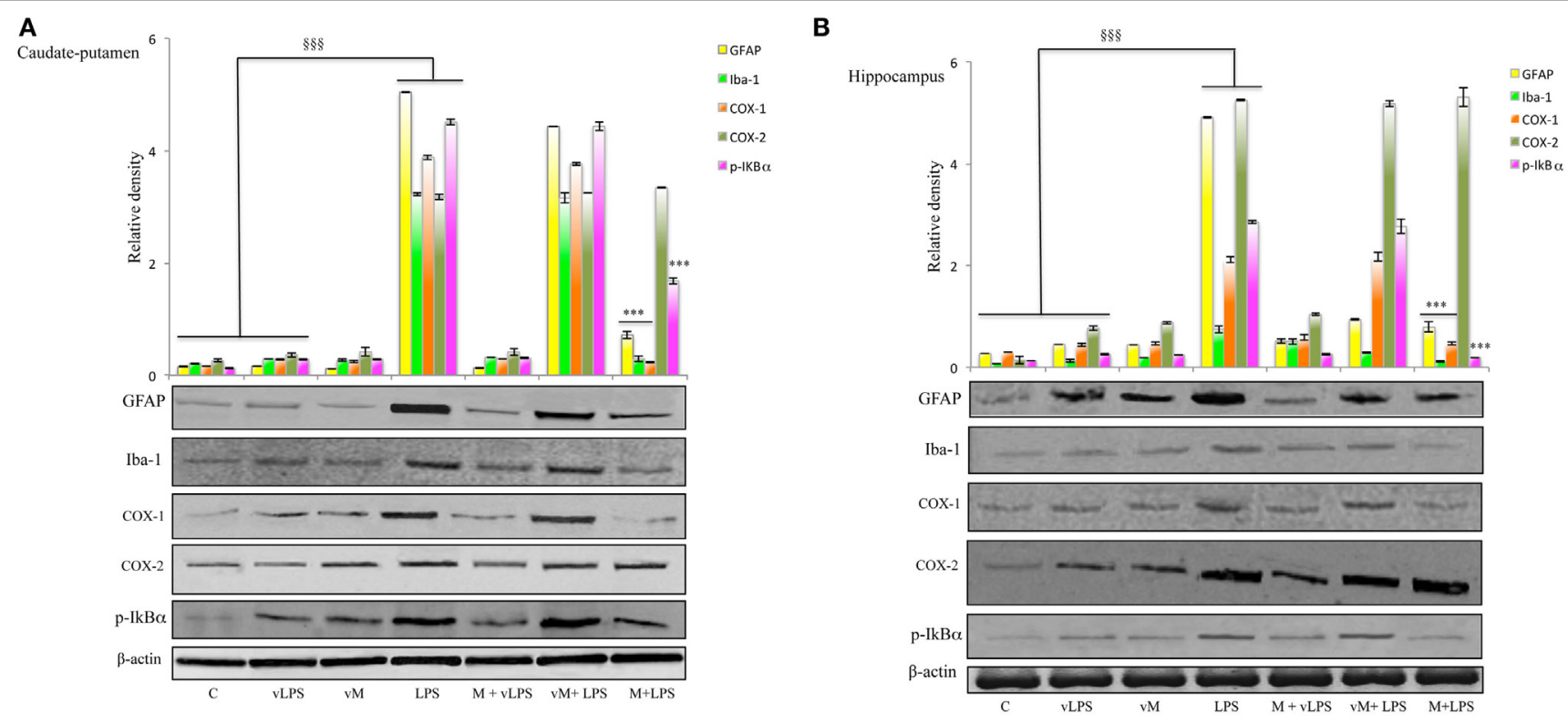

C

D

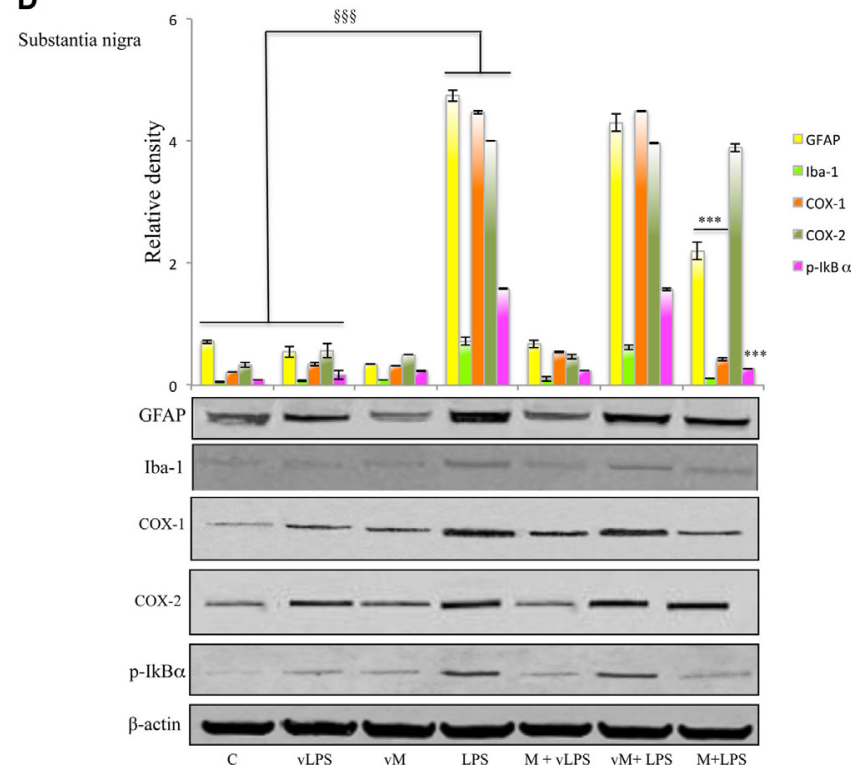

FIGURE 4 | Effect of mofezolac on glial fibrillary acidic protein (GFAP), ionized calcium-binding adapter molecule-1 (Iba-1), cyclooxygenases (COXs), and plkB $\alpha$ expression in (A) caudate-putamen, (B) hippocampus, (C) frontal lobe, and (D) substantia nigra from mice treated with lipopolysaccharide (LPS) alone and LPS in the presence of mofezolac. The quantification of relative band intensities was expressed as relative density, after normalization against $\beta$-actin densitometry. Values represents the means \pm SE of three independent experiments. C, control; vLPS, vehicle of LPS; vM, vehicle of mofezolac; M + vLPS, mofezolac and vehicle of LPS; VM + LPS, vehicle of mofezolac and LPS; M + LPS, mofezolac and LPS. ${ }^{\S \S} p \leq 0.001$ compared with control or vLPS; ${ }^{\star \star \star} p \leq 0.001$ compared with LPS alone.

$\mathrm{p}-\mathrm{I} k \mathrm{~B} \alpha$ levels comparable to controls were detected in mice treated with vLPS + mofezolac, whereas in mice treated with vehicle of mofezolac + LPS, $\mathrm{p}-\mathrm{I} k \mathrm{~B} \alpha$ levels were comparable to those detected in LPS-treated mice (Figure 4).

\section{DISCUSSION}

Clinical data and basic research outcomes showed a strict correlation between neurodegeneration and neuroinflammation $(1,8)$. COXs (both COX-1 and COX-2) play a central role in the inflammatory cascade by converting arachidonic acid (AA) into bioactive prostanoids (19). Both COX isoforms catalyze the same reactions: bis-oxygenation of AA to yield prostaglandin $\mathrm{G}_{2}$ $\left(\mathrm{PGG}_{2}\right)$, and a peroxidase reaction, which converts $\mathrm{PGG}_{2}$ into prostaglandin $\mathrm{H}_{2}\left(\mathrm{PGH}_{2}\right) . \mathrm{PGH}_{2}$ is then transformed into $\mathrm{PGE}_{2}$, $\mathrm{PGF}_{2 \alpha}, \mathrm{PGD}_{2}, \mathrm{PGI}_{2}$, and thromboxane (TX) by specific terminal synthases. In the brain, both COX-1 and COX-2 are constitutively expressed (20), and there are regional differences in the regulation of COXs, probably as a consequence of the different distribution of COX-1 and COX-2 in the cerebral areas (21). 
In physiological conditions, COX-1 is mainly expressed in microglia and perivascular cells, whereas COX-2 is found in postsynaptic dendrites and excitatory terminals, particularly in the cortex, hippocampus, and amygdala, with both neuronal and vascular associations. COX-1 recently has been recognized as a pivotal player in neuroinflammation $(11,22)$. Elevated levels of $\mathrm{TXA}_{2}$ and increased COX-1 expression in the rat hippocampus

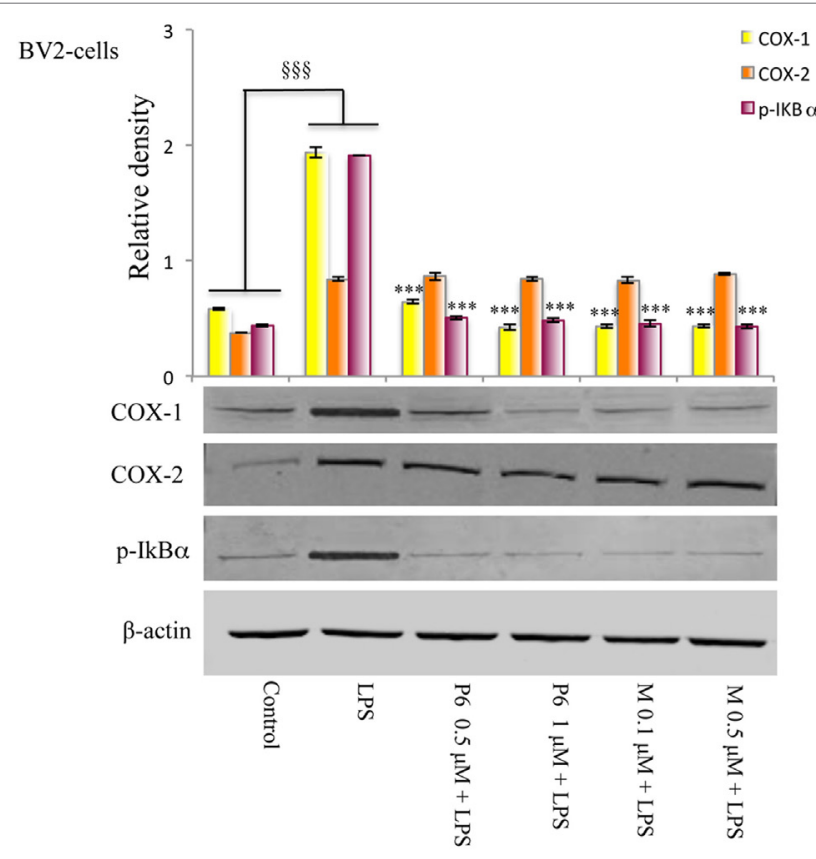

FIGURE 5 | Effects of cyclooxygenase (COX)-1 inhibitors on the COXs expression and NF-kB phosphorylation induced by lipopolysaccharide (LPS) in BV2 microglial cells. Total protein was subjected to SDS-PAGE, followed by immunoblotting using $\mathrm{pl} k \mathrm{~B} \alpha$ antibody. The quantification of relative band intensities was expressed as relative density, after normalization against $\beta$-actin densitometry. Each bar represents the means \pm SE of three independent experiments. BV2 microglial cells incubated with medium alone (control) or treated with LPS alone or in presence of the P6 and mofezolac (M) for 48 h. ${ }^{\S \S} p \leq 0.001$ vs control, ${ }^{\star * *} p \leq 0.001$ vs LPS alone. seem to be correlated to increased brain susceptibility to inflammation and neurodegenerative diseases (23). Moreover, in several models of neuroinflammation, COX-1 has been shown to support inflammatory processes facilitating pro-inflammatory PG upregulation, mainly $\mathrm{PGE}_{2}$ (22).

In this regard, we previously reported that the COX-1 selective inhibitor P6 was able to control the inflammatory response in LPS-treated N13 microglial cells, an in vitro model of neuroinflammation (14). In this context, P6 was able to selectively downregulate COX-1 protein expression, without affecting COX2 levels, as well as reduce $\mathrm{I} k \mathrm{~B} \alpha$ phosphorylation. In this previous study, we proposed a new possible mechanism by which P6 negatively regulates COX-1 expression thereby NF-kB mediated signal-pathway modulation, suggesting the necessity of further studies to validate the efficacy of P6 as well as its analogs in the treatment of neuroinflammatory-based neurodegenerative disease (14).

Since the potential beneficial effect of COX-1 inhibition in the treatment of neuroinflammation has been considered, in the present study, we determined the modulatory effect of two selective COX-1 inhibitors-P6 and mofezolac-both in in vitro and in vivo models of neuroinflammation. For this purpose, we employed the mouse BV-2 microglial cells activated by LPS instead of N13 cell line to exclude a possible bias linked to the in vitro model used. Data of the present study clearly demonstrated that $\mathrm{P} 6$ was able to reduce COX-1 expression and negatively regulate the NF-kB activation, thus showing that these effects are independent from cell line used. Besides, P6 action on LPS-treated BV2 microglial cell responses was compared with the effects of mofezolac, an analgesic drug already approved to be used in humans, exerting its pharmacological action through a highly selective inhibition of COX-1 $(24,25)$. In this regard, mofezolac was able to reduce COX-1 expression in LPS-activated $\mathrm{BV}-2$ cells accompanied to $\mathrm{PGE}_{2}$ release reduction and NF-kB activation downregulation.

More interestingly, these results were confirmed with those derived from the preclinical in vivo model of neuroinflammation, where mice intra-cerebroventricularly received LPS. Concerning in vivo investigation, GFAP and Iba-1 expression, two markers

TABLE 1 | In vitro and in vivo PGE 2 release affected by cyclooxygenase (COX)-1 inhibitors P6 and mofezolac (M).

(A) Effect of COX-1 inhibitors P6 and mofezolac (M) on PGE 2 release ( $\mathrm{ng} / \mathrm{mL}$ ) at $48 \mathrm{~h}$ in lipopolysaccharide (LPS)-stimulated BV2 microglial cells

\begin{tabular}{|c|c|c|c|c|c|}
\hline Control & LPS & $0.5 \mu \mathrm{M}$ P6 + LPS & $1 \mu \mathrm{M}$ P6 + LPS & $0.1 \mu \mathrm{M} M+$ LPS & $0.5 \mu \mathrm{M} M+$ LPS \\
\hline $0.22 \pm 0.025$ & $0.97 \pm 0.021^{a}$ & $0.21 \pm 0.010^{b}$ & $0.12 \pm 0.008^{b}$ & $0.06 \pm 0.004^{b}$ & $0.03 \pm 0.002^{b}$ \\
\hline
\end{tabular}

(B) Effect of mofezolac (M) on $\mathrm{PGE}_{2}$ release ( $\mathrm{ng} / \mathrm{mg}$ tissue) in specimens from LPS-treated mice

\begin{tabular}{|c|c|c|c|c|}
\hline Specimen & Control & Vehicle LPS & LPS & $M+$ LPS \\
\hline Caudate-putamen & $0.15 \pm 0.050$ & $0.73 \pm 0.045^{c}$ & $5.61 \pm 0.136^{c}$ & $1.84 \pm 0.035^{d}$ \\
\hline Hippocampus & $0.31 \pm 0.036$ & $0.69 \pm 0.032^{\circ}$ & $4.55 \pm 0.046^{c}$ & $2.33 \pm 0.032^{d}$ \\
\hline Frontal lobe & $0.33 \pm 0.026$ & $0.68 \pm 0.026^{c}$ & $4.60 \pm 0.055^{c}$ & $2.40 \pm 0.046^{d}$ \\
\hline Substantia nigra & $0.42 \pm 0.021$ & $0.74 \pm 0.059^{\circ}$ & $5.48 \pm 0.115^{c}$ & $3.31 \pm 0.079^{d}$ \\
\hline
\end{tabular}

${ }^{a} p \leq 0.01$ compared with the control value.

${ }^{b} p \leq 0.01$ vs $L P S$ alone

${ }^{c} p \leq 0.001$ vs untreated animals (control).

${ }^{d} p \leq 0.01$ vs LPS-treated animals. 
of neuroinflammation, were reduced by mofezolac to an extent depending on the encephalon area analyzed (see Results). Moreover, the increase of COX-1 expression observed in all the brain sections of LPS-treated mice was selectively downregulated by the in vivo treatment with mofezolac, as well as $\mathrm{PGE}_{2}$ release and $\mathrm{I} k \mathrm{~B} \alpha$ phosphorylation extent assayed in the tested brain areas.

In a previous work of Bosetti et al. carried out on a murine model, it was reported that evident neurodegeneration mediated by activated microglia and increased pro-inflammatory cytokines appeared in the hippocampus after LPS injection (11). Although, previous reports have shown sustained inflammation at $24 \mathrm{~h}$ after LPS injection $(11,26)$, in our in vivo model, we prolonged up to $72 \mathrm{~h}$ the time after LPS intraventricular injection to determine the optimal degree of inflammatory response. In fact, at $72 \mathrm{~h}$ after LPS injection, we clearly detected in all the brain regions tested, represented not only by hippocampus but also caudate, substantia nigra, and frontal lobe, the signs of neuroinflammation in terms of astrocytes and microglia activation.

Hippocampus is a part of the encephalon, located at the temporal lobe, where it plays a crucial role in processing information selected for the long-term memory; therefore, it is important to check what happens at level of this brain area the neuroinflammation modulation (27). Moreover, substantia nigra and caudateputamen, both involved in neurodegenerative processes typically of the Parkinson's disease, as well as frontal lobe, involved in superior cognitive pathways, were investigated to evaluate the effects of COX-1 inhibitors $(28,29)$.

Results from our experiments demonstrated that in vivo treatment with mofezolac was able to reduce glia activation acting selectively on COX-1 expression, thus supporting that COX-1 modulation represents a viable target useful to attenuate the neuroinflammatory response. In this regard, it was reported how COX-1 genetic depletion was able to attenuate microglial and astrocyte activation, reducing pro-inflammatory cytokine expression and preventing the neuronal cells loss in the hippocampus (11).

Recently, it was reported that the potent PET probe highly selective for COX-1, $\left[{ }^{11} \mathrm{C}\right]$-radiolabeled ketoprofen methyl ester was able to detect activated microglia associated with amyloid plaque progression, suggesting the involvement of COX-1 in the neuroinflammatory process in $\mathrm{AD}$ (30). The role of COX-1 in neuroinflammation is also supported by the fact that COX-1 inhibition through drug or gene deletion reduces neuronal damage and inflammatory responses after injection of LPS or A $\beta$ in mouse brain $(31,32)$. The LPS model is particularly relevant to examine activation of brain innate immunity, since it specifically and

\section{REFERENCES}

1. Filiou MD, Arefin AS, Moscato P, Graeber MB. Neuroinflammation differs categorically from inflammation: transcriptomes of Alzheimer's disease, Parkinson's disease, schizophrenia and inflammatory diseases compared. Neurogenetics (2014) 15:201-12. doi:10.1007/s10048-014-0409-x

2. Xu L, He D, Bai Y. Microglia-mediated inflammation and neurodegenerative disease. Mol Neurobiol (2016) 53:6709-15. doi:10.1007/s12035-0159593-4

3. Schafer DP, Lehrman EK, Kautzman AG, Koyama R, Mardinly AR, Yamasaki R, et al. Microglia sculpt postnatal neural circuits in an activity and complement- directly targets microglia, the immune resident cells in the brain, through the CD14 protein binding and subsequent toll-like receptor 4 mediated pro-inflammatory signaling pathway activation. These events cascade culminates to NF-kB activation leading to the release of cytokines, chemokines, ROS, PG, and TX $(33,34)$.

\section{CONCLUSION}

Our results demonstrated that both $\mathrm{P} 6$ and mofezolac were able to reduce COX-1-derived $\mathrm{PGE}_{2}$ release complemented with an antiinflammatory activity at the level of the NF- $\mathrm{kB}$, thus providing mechanistic insights into the suppressive effect of these COX-1 inhibitors on LPS-induced neuroinflammatory response by microglia.

In conclusion, this work consolidated the hypothesis that selective COX-1 inhibitors can positively modify the inflammatory response in LPS-induced neuroinflammatory models. Overall these results, from in vitro and in vivo experiments, indicate the capability of two highly selective COX-1 inhibitors P6 and mofezolac to modulate the NF-kB signaling pathway. These findings emphasize the neuroprotective effect and therapeutic potential of COX-1 inhibitors useful in the control of neuroinflammatory diseases.

\section{ETHICS STATEMENT}

This study was carried out in accordance with the recommendations of Ministero della Salute Decreto Ministeriale, Dott. Fabrizio Bertani. The protocol was approved by the Ministero della Salute Decreto Ministeriale no. 138/2014-B.

\section{AUTHOR CONTRIBUTIONS}

$\mathrm{RC}$ performed in vitro experiments with $\mathrm{AC}$ and RS; DL performed in vivo experiments with FN and LG; MGP designed the work and with PV prepared the tested compounds; GN supervised in vivo experiments; MAP supervised in vitro experiments and wrote the paper; and AS designed the work, supervised all the activities, and wrote the paper with help from other authors.

\section{ACKNOWLEDGMENTS}

This work was supported by a grant from the Italian "Ministero dell'Istruzione, dell'Università e della Ricerca” (MIUR) under the project entitled "Research, Application, Innovation, Services in Bioimaging (R.A.I.S.E. in Bioimaging)," code PON01_03054. First AIRC Grant-MFAG2015 (Project Id. 17566).

dependent manner. Neuron (2012) 74:691-705. doi:10.1016/j.neuron.2012. 03.026

4. Hughes V. Microglia: the constant gardeners. Nature (2012) 485:570-2. doi:10.1038/485570a

5. Gemma C, Bachstetter AD. The role of microglia in adult hippocampal neurogenesis. Front Cell Neurosci (2013) 7:229. doi:10.3389/fncel.2013.00229

6. Nakanishi H, Hayashi Y, Wu Z. The role of microglial mtDNA damage in age-dependent prolonged LPS-induced sickness behavior. Neuron Glia Biol (2011) 7:17-23. doi:10.1017/S1740925X1100010X

7. Choi DK, Koppula S, Suk K. Inhibitors of microglial neurotoxicity: focus on natural products. Molecules (2011) 16:1021-43. doi:10.3390/molecules16021021 
8. Glass CK, Saijo K, Winner B, Marchetto MC, Gage FH. Mechanisms underlying inflammation in neurodegeneration. Cell (2010) 140:918-34. doi:10.1016/j.cell.2010.02.016

9. Ransohoff RM, Perry VH. Microglial physiology: unique stimuli, specialized responses. Annu Rev Immunol (2009) 27:119-45. doi:10.1146/annurev. immunol.021908.132528

10. Cacciatore I, Marinelli L, Fornasari E, Cerasa LS, Eusepi P, Türkez H, et al. Novel NSAID-derived drugs for the potential treatment of Alzheimer's disease. Int J Mol Sci (2016) 17:E1035. doi:10.3390/ijms17071035

11. Choi SH, Aid S, Bosetti F. The distinct roles of cyclooxygenase-1 and -2 in neuroinflammation: implications for translational research. Trends Pharmacol Sci (2009) 30:174-81. doi:10.1016/j.tips.2009.01.002

12. Vitale P, Scilimati A, Perrone MG. Update on SAR studies toward new COX-1 selective inhibitors. Curr Med Chem (2015) 22:4278-92. doi:10.2174/092986 7322666151029104717

13. Vitale P, Panella A, Scilimati A, Perrone MG. COX-1 inhibitors: beyond structure toward therapy. Med Res Rev (2016) 36:641-71. doi:10.1002/med.21389

14. Calvello R, Panaro MA, Carbone ML, Cianciulli A, Perrone MG, Vitale P, et al. Novel selective COX-1 inhibitors suppress neuroinflammatory mediators in LPS-stimulated N13 microglial cells. Pharmacol Res (2012) 65:137-48. doi:10.1016/j.phrs.2011.09.009

15. Di Nunno L, Vitale P, Scilimati A, Tacconelli S, Patrignani P. Novel synthesis of 3,4-diarylisoxazole analogues of valdecoxib: reversal cyclooxygenase-2 selectivity by sulfonamide group removal. J Med Chem (2004) 47:4881-90. doi:10.1021/jm040782x

16. Micetich RG. 3,4-Diarylisoxazol-5-Acetic Acid Compounds, Process for Preparing the Same, and Pharmaceuticals Containing the Same. European patent EP 0026928 A1 (1981).

17. Bi W, Zhu L, Jing X, Zeng Z, Liang Y, Xu A, et al. Rifampicin improves neuronal apoptosis in LPS-stimulated co-cultured BV2 cells through inhibition of the TLR-4 pathway. Mol Med Rep (2014) 10:1793-9. doi:10.3892/mmr.2014.2480

18. Haw RT, Tong CK, Yew A, Lee HC, Phillips JB, Vidyadaran S. A three-dimensional collagen construct to model lipopolysaccharide-induced activation of BV2 microglia. J Neuroinflammation (2014) 11:134-44. doi:10.1186/1742-209411-134

19. Morita I. Distinct functions of COX-1 and COX-2. Prostaglandins Other Lipid Mediat (2002) 68:165-75. doi:10.1016/S0090-6980(02)00029-1

20. Aïd S, Bosetti F. Targeting cyclooxygenases-1 and -2 in neuroinflammation: therapeutic implications. Biochimie (2011) 93:46-51. doi:10.1016/j. biochi.2010.09.009

21. Yasojima K, Schwab C, McGeer EG, McGeer PL. Distribution of cyclooxygenase-1 and cyclooxygenase-2 mRNAs and proteins in human brain and peripheral organs. Brain Res (1999) 830:226-36. doi:10.1016/S0006-8993(99) 01389-X

22. Matousek SB, Hein AM, Shaftel SS, Olschowka JA, Kyrkanides S, O’Banion MK. Cyclooxygenase-1 mediates prostaglandin E(2) elevation and contextual memory impairment in a model of sustained hippocampal interleukin-1beta expression. J Neurochem (2010) 114:247-58. doi:10.1111/j.1471-4159.2010.06759.x

23. Aïd S, Silva AC, Candelario-Jalil E, Choi SH, Rosenberg GA, Bosetti FJ. Cyclooxygenase-1 and -2 differentially modulate lipopolysaccharide-induced blood-brain barrier disruption through matrix metalloproteinase activity. J Cereb Blood Flow Metab (2010) 30:370-80. doi:10.1038/jcbfm.2009.223
24. Goto K, Ochi H, Yasunaga Y, Matsuyuki H, Imayoshi T, Kusuhara H, et al. Analgesic effect of mofezolac, a non-steroidal anti-inflammatory drug, against phenylquinone-induced acute pain in mice. Prostaglandins Other Lipid Mediat (1998) 56:245-54. doi:10.1016/S0090-6980(98)00054-9

25. Abdelazeem AH, El-Saadi MT, Safi El-Din AG, Omar HA, El-Moghazy SM. Design, synthesis and analgesic/anti-inflammatory evaluation of novel diarylthiazole and diarylimidazole derivatives towards selective COX-1 inhibitors with better gastric profile. Bioorg Med Chem (2017) 25:665-76. doi:10.1016/j. bmc.2016.11.037

26. Milatovic D, Zaja-Milatovic S, Montine KS, Horner PJ, Montine TJ Pharmacologic suppression of neuronal oxidative damage and dendritic degeneration following direct activation of glial innate immunity in mouse cerebrum. J Neurochem (2003) 87:1518-26. doi:10.1046/j.1471-4159.2003.02120.x

27. Barrientos RM, Kitt MM, Watkins LR, Maier SF. Neuroinflammation in the normal aging hippocampus. Neuroscience (2015) 309:84-99. doi:10.1016/j. neuroscience.2015.03.007

28. Lofrumento DD, Saponaro C, Cianciulli A, De Nuccio F, Mitolo V, Nicolardi G, et al. MPTP-induced neuroinflammation increases the expression of proinflammatory cytokines and their receptors in mouse brain. Neuroimmunomod (2011) 18:79-88. doi:10.1159/000320027

29. Lim A, Krajina K, Marsland AL. Peripheral inflammation and cognitive aging Mod Trends Pharmacopsychiatry (2013) 28:175-87. doi:10.1159/000346362

30. Choi SH, Bosetti F. Cyclooxygenase-1 null mice show reduced neuroinflammation in response to beta-amyloid. Aging (Albany NY) (2009) 1:234-44. doi:10.18632/aging.100021

31. Choi SH, Langenbach R, Bosetti F. Genetic deletion or pharmacological inhibition of cyclooxygenase-1 attenuate lipopolysaccharide-induced inflammatory response and brain injury. FASEB J (2008) 22:1491-501. doi:10.1096/ fj.07-9411com

32. Shukuri M, Mawatari A, Ohno M, Suzuki M, Doi H, Watanabe Y, et al. Detection of cyclooxygenase- 1 in activated microglia during amyloid plaque progression: PET studies in Alzheimer's disease model mice. J Nucl Med (2016) 57:291-6. doi:10.2967/jnumed.115.166116

33. Aid S, Bosetti F. Targeting cyclooxygenases-1 and -2 in neuroinflammation: Therapeutic implications. Biochimie (2011) 93:46-51. doi:10.1016/j. biochi.2010.09.009

34. Janova H, Böttcher C, Holtman IR, Regen T, van Rossum D, Götz A, et al. CD14 is a key organizer of microglial responses to CNS infection and injury. Glia (2016) 64:635-49. doi:10.1002/glia.22955

Conflict of Interest Statement: The authors declare that the research was conducted in the absence of any commercial or financial relationships that could be construed as a potential conflict of interest.

Copyright (C) 2017 Calvello, Lofrumento, Perrone, Cianciulli, Salvatore, Vitale, De Nuccio, Giannotti, Nicolardi, Panaro and Scilimati. This is an open-access article distributed under the terms of the Creative Commons Attribution License (CC BY). The use, distribution or reproduction in other forums is permitted, provided the original author(s) or licensor are credited and that the original publication in this journal is cited, in accordance with accepted academic practice. No use, distribution or reproduction is permitted which does not comply with these terms. 